\section{Lungenfunktion: Risikofaktoren für Veränderungen im Alter}

Luoto J et al. Relative and absolute lung function change in a general population aged 60-102 years. Eur Respir ] 2019; doi:10.1183/ 13993003.01812-2017

Obwohl es heute zahlreiche Studien über die Abnahme der Lungenfunktion bei COPD-Patienten gibt, sind Details über entsprechende Veränderungen in der alternden Normalbevölkerung bisher weitestgehend unbekannt. Luoto und Team haben hier angesetzt und im Rahmen einer Längsschnittstudie die Verläufe von FEV $_{1}$ und der Vitalkapazität bei Schweden zwischen 60 und 102 Jahren analysiert.

Bei Patienten mit chronisch obstruktiver Lungenerkrankung (COPD) nehmen spirometrische Parameter wie das forcierte endexspiratorische Volumen in der ersten Sekunde $\left(\mathrm{FEV}_{1}\right)$ oder die forcierte Vitalkapazität (fVC) im Krankheitsverlauf tendenziell $a b$. Wie sich diese beiden wichtigen Parameter allerdings in der alternden Normalbevölkerung entwickeln, darüber gibt es bislang nur wenige Anhaltspunkte. Luoto und Kollegen haben sich diesem Thema nun angenommen und im Rahmen einer groß angelegten schwedischen Kohortenstudie die absolute und relative $\mathrm{FEV}_{1}$ und $\mathrm{FVC}$ in der alternden Bevölkerung zwischen 60 und 102 Jahren im prospektiven Längsschnittdesign untersucht.

Sie stellten zuvor die Hypothese auf, dass weibliches Geschlecht, Bildungsniveau, Entzündungsparameter wie CRP und Raucherstatus mit etwaigen Veränderungen bzw. einer Abnahme der Werte im Alter zusammenhängen würden. Weiterhin wollten sie herausfinden, ob es Unterschiede zwischen den relativen und absoluten Veränderungen beider Parameter gibt.

Geeignete Probanden aus dem Schwedischen Nationalregister wurden zunächst kontaktiert und um die Teilnahme gebeten. Eingeschlossene Schweden stellten sich dann im Laufe einer Nachbeobach- tungszeit von durchschnittlich 13,5 Jahren regelmäßig zur spirometrischen Untersuchung und Befragung vor.

Zusätzlich registrierten die Forscher für jeden Studienteilnehmer Information über das Bildungsniveau, soziodemografische Daten, Erkrankungen und Entzündungsparameter und führten verschiedene Test zum kognitiven Leistungsniveau wie den Mini-mental-statusTest (MMST) durch. Schließlich rechneten die Autoren eine Regressionsanalyse in verschiedenen Varianten, um potenzielle Einflussfaktoren nachweisen zu können.

\section{Geschlecht spielt eine Rolle}

4459 Menschen nahmen an der Studie teil, 55,8\% von ihnen waren weiblich. Das durchschnittliche Alter konnte auf 70,6 Jahre mit einer Standardabweichung von 10,6 Jahren beziffert werden. 17,3\% der Teilnehmer waren zum Studienzeitpunkt aktive Raucher, 3,1\% gaben einen niedrigen sozioökonomischen Status einschließlich Bildungsniveau an. Die häufigsten Morbiditäten waren Bluthochdruck mit 30,4\%, Diabetes Typ 2 (7,3\%) und koronare Herzerkrankung (16,4\%). Bei 3,1\% aller Teilnehmer konnten die Forscher einen erhöhten CRP-Wert von über $20 \%$ der Norm feststellen, 9,1\% der Teilnehmer litten an einer relevanten Anämie.

3736 aller zunächst rekrutierten Teilnehmer absolvierten schließlich während der 13,5-jährigen Nachbeobachtungszeit zwischen einer und 5 spirometrischen Untersuchungen. Die bereinigte absolute Veränderung lag bei - 51,7 ml für die $\mathrm{FEV}_{1}$ und bei $-56,25 \mathrm{ml}$ für die fVC. Die bereinigte relative Veränderung pro Jahr betrug $-2,97 \%$ für die $\mathrm{FEV}_{1}$ und $-2,46 \%$ für die fVC. Risikofaktoren für einen erhöhten relativen $\mathrm{fVC}$ - und $\mathrm{FEV}_{1}$-Rückgang waren weibliches Geschlecht, niedriges Bildungsniveau, höheres Alter, Raucherstatus sowie ein erhöhter CRP-Wert.

Letzterer war allerdings für die $\mathrm{FEV}_{1}$ nicht signifikant. Für einen erhöhten absoluten Rückgang waren die Risikofaktoren hin- gegen männliches Geschlecht, positiver Raucherstatus $\left(\mathrm{FEV}_{1}\right.$ ) und niedriges Bildungsniveau (fVC). Relative, aber nicht die absoluten Veränderungen korrelierten signifikant mit klinisch relevanten Funktionsmarkern wie dem MMST. Insgesamt betrachten die Forscher auf Grundlage ihrer Resultate die Eingangshypothese für bestätigt und planen weitere Untersuchungen zum Thema.

\section{FAZIT}

In dieser nationalen Kohortenstudie gingen die Risikofaktoren weibliches Geschlecht, positiver Raucherstatus, Inflammation und niedriges Bildungsniveau mit einer relativen Abnahme

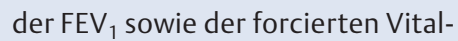
kapazität bei Menschen zwischen 60 und 102 Jahren einher. Da Geschlecht, Bildung und Raucheranamnese ebenfalls einen relevanten Einfluss auf die Abnahme der absoluten Werte hatten, empfehlen die Autorinnen/Autoren eine stärkere Beachtung und $\mathrm{Er}$ forschung dieser Zusammenhänge.

Dipl.-Psych. Annika Simon, Hannover 